\title{
The many ways of osteoclast activation
}

\author{
Joseph Lorenzo
}

Departments of Medicine and Orthopaedics, UConn Health, Farmington, Connecticut, USA

\begin{abstract}
Osteoclasts are the cells responsible for bone resorption, a process that is essential for the maintenance of healthy bones. Bone diseases, such as osteoporosis, which are characterized by high rates of bone resorption and loss of bone mass, may benefit from treatments that inhibit osteoclast formation and/or function. The RANKL/RANK pathway is critical for both osteoclast formation and function, and these effects are thought to be mediated by the transcription factor nuclear factor of activated T cells, cytoplasmic 1 (NFATc1). In this issue of the JCI, Bae et al. challenge the convention that NFATC1 is the sole critical regulator of RANKL/RANKdependent osteoclast activation. Specifically, the authors show that MYC drives metabolic reprogramming in osteoclasts and that MYC induces estrogen receptor-related receptor $\alpha(E R R \alpha)$ to regulate osteoclastogenesis. Importantly, both loss of MYC and pharmacological inhibition of ERR $\alpha$ attenuated bone loss in a mouse model of osteoporosis. Together, the results of this study suggest that the MYC/ERR $\alpha$ pathway should be further explored as a drug target for bone diseases.
\end{abstract}

\section{Osteoclasts in bone health and disease}

Osteoclasts, which are essential for normal bone health, are multinucleated giant cells that mediate bone resorption (1). These cells originate from hematopoietic, myeloid, multipotential precursors that differentiate into mature osteoclasts, primarily through interactions with two cytokines: macrophage-CSF (M-CSF, also known as, CSF-1) and RANKL (2). RANKL is necessary for osteoclast formation and function, as mice lacking RANKL or its cognate cell-surface receptor, RANK, fail to form osteoclasts or resorb bone under homeostatic conditions $(3,4)$. Whether osteoclasts can form in the absence of RANKL/RANK signaling in vivo during states of inflammation or malignancy in bone remains controversial $(5,6)$. The most common bone disease is osteoporosis, which affects more than 8 million Americans, accounts for approximately 1.6 million fractures yearly, and has an annual cost to the US health care system of 22 billion dollars (7). Osteoporosis develops when the rates of bone resorption exceed those of bone formation, causing low bone mass and an increased risk of fragility fractures (8). Current therapies for this condition and other metabolic bone diseases that are associated with excessive resorption confer a risk for severe, albeit rare, adverse effects that limit the use of these treatments (9). Hence, there is a substantial need to fully understand the mechanisms by which RANKL stimulates osteoclast-mediated bone resorption in order to identify novel drug targets that can be exploited to develop better therapies for these conditions.

A number of molecules have been identified as downstream mediators of RANK/ RANKL signaling in osteoclasts and osteoclast precursors. These include TNF receptor-associated factor 6 (TRAF6), NF- $\mathrm{kB}$, MAPK, and activator protein 1 (AP-1) (10), all of which are costimulated by immunoreceptor tyrosine-based activation motif

\section{Related Article: p. 2555}

Conflict of interest: J. Lorenzo has received research support from Boehringer Ingelheim Pharmaceuticals Inc. Reference information: / Clin Invest. 2017;127(7):2530-2532. https://doi.org/10.1172/JCI94606.
(ITAM) signaling (11). These factors downstream of RANKL/RANK are thought to influence osteoclasts through their effects on a common transcription factor, nuclear factor of activated $\mathrm{T}$ cells, cytoplasmic 1 (NFATc1) $(12,13)$. Several lines of evidence point to a critical role of NFATc1 in osteoclast formation and function. Specifically, NFATc1-deficient embryonic stem cells cannot differentiate into osteoclasts, ectopic expression of NFATc1 in osteoclast precursors results in their differentiation into mature cells in the absence of RANKL, and targeted disruption of $\mathrm{Nfatcl}$ in hematopoietic cells in mice increases bone mass, with a marked decrease in osteoclasts $(12,13)$.

In this issue, Bae et al. provide data to challenge the view that NFATc1 is the sole critical transcription factor responsible for mediating RANKL responses in osteoclasts (14). The transcription factor MYC is well described to have multiple effects on cellular differentiation and proliferation in a variety of cell types (15). Bae and colleagues examined whether MYC has a similar role in osteoclasts. It has been known for fifteen years that MYC does influence RANKL-induced effects on osteoclasts. RAW 264.7 cells are a transformed murine myeloid cell line that can be driven into an osteoclast-like phenotype by treatment with RANKL. Battaglino et al. (16) showed that RANKL treatment of RAW 264.7 cells stimulated the expression of MYC and that MYC inhibition blocked the ability of these cells to resorb bone. Subsequently, Daumer et al. (17) demonstrated that MYC influences the activity of the tartrate-resistant acid phosphatase promoter, which is an important gene for osteoclast function. More recently, Indo et al. (18) demonstrated in osteoclasts that MYC influences glutamine metabolism (glutaminolysis), which is used by cells to generate energy for metabolic pathways.

\section{MYC contributes to RANKL/ RANK signaling}

Members of the Bae et al. group previously demonstrated that MYC inhibition abrogates RANKL-induced NFATc1 


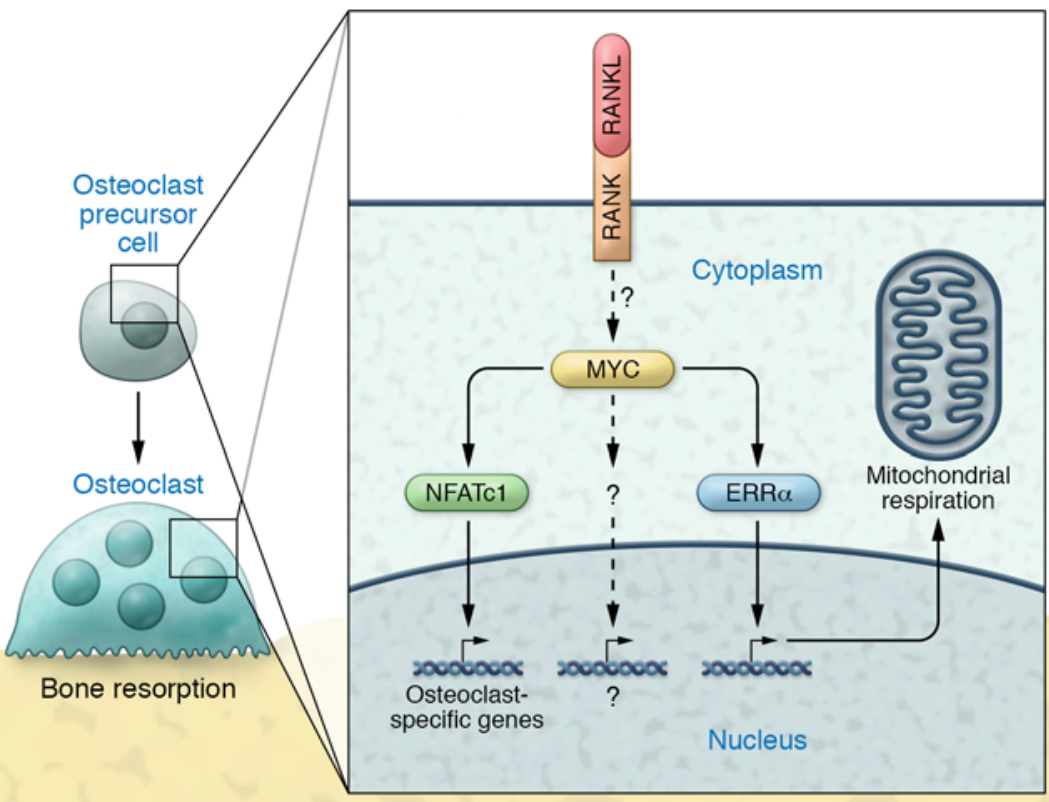

Figure 1. Mechanisms of osteoclast activation. In both osteoclast precursor cells and mature osteoclasts, RANKL/RANK signaling stimulates MYC expression through as-yet undefined mechanisms. MYC, in turn, stimulates the production of ERR $\alpha$ and NFATC1 and may also affect additional pathways. All of these signals ultimately produce multinucleated osteoclasts that can resorb bone.

expression and RANKL-stimulated recruitment of MYC to the Nfatc1 promoter (19). Hence, they hypothesized that MYC is an upstream signal utilized by RANKL-RANK interaction at the cell surface to enhance NFATc1 production and stimulate osteoclast formation and function. Now, these authors have studied a mouse model in which MYC was conditionally deleted in myeloid lineage cells (MYC ${ }^{\Delta \mathrm{M}}$ mice). Not surprisingly, given their previous data demonstrating that MYC is an upstream signal that mediates RANKL-induced NFATc1 expression, $\mathrm{MYC}^{\Delta \mathrm{M}}$ mice had increased bone mass and decreased osteoclasts, with a normal number of bone-forming osteoblasts. In vitro, cultured bone marrow osteoclast precursor cells (OCPs) from $\mathrm{MYC}^{\Delta \mathrm{M}}$ mice completely failed to form mature osteoclasts in response to RANKL and M-CSF and had decreased NFATc1 expression. However, transduction of MYC-deficient OCPs with a conditionally active NFATc1 construct did not rescue osteoclastogenesis in these cells. This unexpected result argues that additional, NFATc1-independent pathways downstream of MYC mediate the ability of RANKL/RANK signaling to influence osteoclasts.
Bae et al. performed transcriptomic analysis of RANKL-treated WT and $\mathrm{MYC}^{\Delta \mathrm{M}}$ cells to identify additional pathways downstream of RANKL and MYC. This analysis revealed that the expression of genes associated with metabolic pathways, such as the TCA cycle and oxidative phosphorylation, were MYC dependent, as they were induced by RANKL in WT, but not MYC ${ }^{\Delta \mathrm{M}}$, cells. Furthermore, oxygen consumption, ATP production, and respiratory capacity and reserve were decreased in $\mathrm{MYC}^{\Delta \mathrm{M}}$ cells, with no difference in mitochondrial mass between WT and MYC ${ }^{\Delta \mathrm{M}}$ cells. These findings are complementary to the work of Nishikawa et al. (20), who previously described an important role of mitochondrial respiration in osteoclast differentiation.

Bae et al. subsequently used gene set enrichment analysis to identify transcription factor-binding motifs that were enriched in the promoters of the MYC-dependent genes identified in their transcriptomic analysis. This enrichment revealed estrogen-related receptor $\alpha(\operatorname{ERR} \alpha)$ to be a MYC-dependent transcription factor with binding motifs within the first $2 \mathrm{~kb}$ of MYC-regulated gene promoters. Additionally, RANKL stimulated ERR $\alpha$ expression in WT, but not $\mathrm{MYC}^{\Delta \mathrm{M}}$ cells, and
ChIP analysis showed that RANKL treatment recruits MYC to the Esrra promoter. Moreover, MYC expression did not rescue osteoclastogenesis in $\mathrm{MYC}^{\Delta \mathrm{M}}$ cells in which $\mathrm{ERR} \alpha$ was inhibited with the small-molecule inhibitor XCT790. Additional studies showed that the effects of RANKL on respiration were lost when ERR $\alpha$ was inhibited or deficient. Finally, coexpression of conditionally activated NFATc1 and ERR $\alpha$ in MYC-deficient OCP cultures partially rescued osteoclastogenesis. In total, these results demonstrate that ERR $\alpha$ mediates some of the effects of MYC on osteoclasts and synergizes with NFATc1 to mediate RANKL-stimulated bone resorption.

Bae et al. also evaluated the effect of the MYC/ERR $\alpha$ pathway on in vivo osteoclastogenesis and resorption (14). Specifically, the authors used the well-described ovariectomy model in mice to produce estrogen deficiency, which mimics postmenopausal osteoporosis and enhances osteoclastogenesis and bone resorption rates (21). Compared with ovariectomized WT mice, ovariectomized $\mathrm{MYC}^{\Delta \mathrm{M}}$ mice had considerably less bone loss. Importantly, treatment of ovariectomized WT mice with the ERR $\alpha$ inhibitor XCT790 reduced bone loss, although, not to the same degree as that seen in $\mathrm{MYC}^{\Delta \mathrm{M}}$ mice.

\section{Concluding remarks}

Taken together, the studies by Bae and colleagues (14) demonstrate that MYC functions as an important molecule mediating RANKL/RANK signaling in osteoclasts through mechanisms that are both dependent on and independent of NFATc1 (Figure 1). Hence, these results argue that NFATc1 is not the only critical regulator of RANKL/ RANK-mediated signaling in osteoclasts. Additionally, this study shows that RANKL/ RANK-mediated MYC signaling has significant effects on osteoclast energy metabolism, which is essential for these cells to perform the work of bone resorption. Most important, these studies identify the MYC/ ERR $\alpha$ pathway as a potential drug target for the development of novel therapies to treat metabolic bone diseases caused by excessive osteoclast activity.

\section{Acknowledgments}

This work was supported by grant R01AR063661 from the National Institute of Arthritis and Musculoskeletal 
and Skin Diseases of the NIH and by a research grant from Boehringer Ingelheim Pharmaceuticals Inc.

Address correspondence to: Joseph Lorenzo, MC5456, UConn Health, 263 Farmington Avenue, Farmington, Connecticut 06030-5456, USA. Phone: 860.679.8199; Email: jlorenzo@uchc.edu.

1. Lorenzo J, Horowitz M, Choi Y. Osteoimmunology: interactions of the bone and immune system. Endocr Rev. 2008;29(4):403-440.

2. Jacome-Galarza CE, Lee SK, Lorenzo JA, Aguila HL. Identification, characterization, and isolation of a common progenitor for osteoclasts, macrophages, and dendritic cells from murine bone marrow and periphery. J Bone Miner Res. 2013;28(5):1203-1213.

3. Kong YY, et al. OPGL is a key regulator of osteoclastogenesis, lymphocyte development and lymph-node organogenesis. Nature. 1999;397(6717):315-323.

4. Dougall WC, et al. RANK is essential for osteoclast and lymph node development. Genes Dev. 1999;13(18):2412-2424.

5. Tanaka S. RANKL-Independent Osteoclastogenesis: A Long-Standing Controversy. J Bone Miner Res. 2017;32(3):431-433.
6. Fujiwara Y, Piemontese M, Liu Y, Thostenson JD, Xiong J, O'Brien CA. RANKL (Receptor Activator of NFKB Ligand) produced by osteocytes is required for the increase in $\mathrm{B}$ cells and bone loss caused by estrogen deficiency in mice. J Biol Chem. 2016;291(48):24838-24850.

7. Blume SW, Curtis JR. Medical costs of osteoporosis in the elderly Medicare population. Osteoporos Int. 2011;22(6):1835-1844.

8. Lorenzo JA, Canalis E, Raisz LG. Metabolic Bone Disease. In: Kronenberg H, Melmed S, Polonsky KS, Larsen PR, eds. Williams Text Book of Endocrinology. 11th ed. Philadelphia, Pennsylvania, USA: Saunders-Elsevier; 2008:1269-1310.

9. Khosla S, Shane E. A crisis in the treatment of osteoporosis. JBone Miner Res. 2016;31(8):1485-1487.

10. Nakashima T, Takayanagi $\mathrm{H}$. New regulation mechanisms of osteoclast differentiation. Ann N Y Acad Sci. 2011;1240:E13-E18.

11. Humphrey MB, Nakamura MC. A Comprehensive Review of Immunoreceptor Regulation of Osteoclasts. Clin Rev Allergy Immunol. 2016;51(1):48-58.

12. Takayanagi $\mathrm{H}$, et al. Induction and activation of the transcription factor NFATc1 (NFAT2) integrate RANKL signaling in terminal differentiation of osteoclasts. Dev Cell. 2002;3(6):889-901.

13. Aliprantis AO, et al. NFATc1 in mice represses osteoprotegerin during osteoclastogenesis and dissociates systemic osteopenia from inflammation in cherubism. J Clin Invest. 2008;118(11):3775-3789.

14. Bae S, et al. MYC-dependent oxidative metabolism regulates osteoclastogenesis via nuclear receptor ERR $\alpha$. J Clin Invest. 2017;127(7):2555-2568.

15. Gnanaprakasam JN, Wang R. MYC in regulating immunity: metabolism and beyond. Genes (Basel). 2017;8(3):E88.

16. Battaglino R, Kim D, Fu J, Vaage B, Fu XY, Stashenko $P$. c-myc is required for osteoclast differentiation. JBone Miner Res. 2002;17(5):763-773.

17. Daumer KM, Taparowsky EJ, Hall DJ, Steinbeck MJ. Transcription from the tartrate-resistant acid phosphatase promoter is negatively regulated by the Myc oncoprotein. J Bone Miner Res. 2002;17(9):1701-1709.

18. Indo Y, et al. Metabolic regulation of osteoclast differentiation and function.JBone Miner Res. 2013;28(11):2392-2399.

19. Park-Min KH, et al. Inhibition of osteoclastogenesis and inflammatory bone resorption by targeting BET proteins and epigenetic regulation. Nat Commun. 2014;5:5418

20. Nishikawa K, et al. DNA methyltransferase 3a regulates osteoclast differentiation by coupling to an S-adenosylmethionine-producing metabolic pathway. Nat Med. 2015;21(3):281-287.

21. Komori T. Animal models for osteoporosis. Eur J Pharmacol. 2015;759:287-294. 